\title{
Toying with the Toolbox: How Metaphysics Can Still Make a Contribution
}

\author{
Steven French ${ }^{1}$
}

Published online: 5 February 2018

(C) The Author(s) 2018. This article is an open access publication

\begin{abstract}
Current analytic metaphysics has been claimed to be, at best, out of touch with modern physics, at worst, actually in conflict with the latter (Callender, in: French, Saatsi (eds) The continuum companion to the philosophy of science, Continuum, London, 2011; Ladyman and Ross Every thing must go: metaphysics naturalized, Oxford University Press, Oxford, 2007). While agreeing with some of these claims, it has been suggested that metaphysics may still be of service by providing a kind of 'toolbox' of devices that philosophers of science can access in order to help provide an interpretation of theories in fundamental physics (French and McKenzie in Eur J Anal Philos 8:42-59, 2012; see also French and McKenzie, in: Bigaj, Wuthrich (eds) Metaphysics in contemporary physics, Rodopi, Amsterdam, 2015). In this context it has been argued that 'standard' forms of dispositionalism simply cannot be sustained in the context of modern physics but that certain 'non-standard' views may provide the resources to help explicate the sense in which physics may be regarded as 'modally informed'. Here that suggestion will be further extended in order to consider the implications both with regard to the overall relevance of metaphysics given advances in science and for the prospects of a naturalised metaphysics more generally. In particular, this paper will focus on three concerns: (1) that the particular tools identified are not, in fact, 'scientifically disinterested' and thus that the distinction between 'naturalised' and 'non-naturalised' metaphysics is at best vague or poorly drawn; (2) that the usefulness of such tools depends on their being shaped to fit the relevant physics and thus the latter 'guts' metaphysics; (3) that if metaphysics does prove to be useful in this sense then we have no reason to scorn non-naturalised metaphysics to begin with.
\end{abstract}

Keywords Dispositionalism - Identity of indiscernibles · Metaphysics · Physics · Potentiality · Standard Model · Symmetry · Toolbox

Steven French

S.R.D.French@leeds.ac.uk

1 School of Philosophy, Religion and History of Science (School of PRHS), University of Leeds, Leeds LS2 9JT, UK 
A great deal has been written recently on the relationship between metaphysics and science. To a significant degree, the current debate was sparked by Ladyman and Ross's provocative claims that "mainstream metaphysics... floats entirely free of science" and as such ought to be "discontinued." (Ladyman and Ross 2007; for similar sentiments, see also Callender 2011). Such claims have been supported in a variety of ways but in particular via surveys of metaphysicians' analyses of, for example, the nature of objects-and more particularly, how they compose and are composed. It is then argued that these analyses at best appeal to a kind of crude atomism of the sort that has long since been supplanted and, more generally, appear to lie at some remove from pertinent developments in modern science.

While broadly agreeing with this negative appraisal of the current state of metaphysics, and, in particular, with the claim that the implications of modern physics impact significantly on many paradigmatic metaphysical claims, it has been argued that something positive can yet be retrieved from this debate, in that such 'mainstream' and, typically, scientifically disinterested metaphysics can still serve an instrumental purpose in acting as a kind of 'toolbox' for philosophers of science (French and McKenzie 2012). The core idea is that despite being so removed from modern science, such metaphysics can yet provide a range of devices and manoeuvres that philosophers of science, and philosophers of physics in particular, can deploy in articulating the implications of modern physics, in particular, for our view of the world (for further on this deployment in general, see French forthcoming and for its impact on scientific realism, see French 2018).

\section{The Implications of Physics}

The core claim of the above argument is thus two-fold:

(1) If metaphysics is to be taken to have something direct to say about reality, the implications of physics need to be properly appreciated and this will have implications for certain 'paradigmatic' metaphysical positions.

(2) However, one does not have to agree with the claim that aprioristic metaphysics should be 'discontinued', since various considerations suggest that we should value scientifically disinterested metaphysics as a 'toolbox' for philosophers of science.

Let us begin with claim (1) and the issue of how the implications of physics might be appreciated. We can take these implications to have either a positive or a negative impact, broadly understood. Thus the impact might be understood as positive in the sense that metaphysical claims might be taken to be supported by virtue of the (empirical) success of theories to which they are related (see in particular Hawley 2006); conversely, it could be understood as negative in the sense that certain claims might be seen as undermined and hence rejected by virtue of that success. Let us consider each of these in turn.

In order to motivate the first claim, take, for example, that old chestnut, the Principle of Identity of Indiscernibles (PII). Not for nothing did Leibniz send Princess Sophie running off through the Hohenzollern gardens to look for two leaves that might appear exactly alike but which on closer inspection would always manifest some difference-the Principle seems supported by our observations of everyday objects. And we can go further: if we allow spatio-temporal properties to be included within the scope of the principle, then it appears to be supported by classical physics also: two electrons, say, classically understood, will have all state-independent properties (charge, mass and so forth) in common and but will differ in their spatio-temporal properties (assuming impenetrability; see 
French and Krause 2006). It is precisely this support that has led certain metaphysicians to insist that although the Principle could not be necessarily true (given the famous possible world counterexamples; see Black 1952), it must surely be contingently so (Casullo 1984).

Now, of course, one might probe a little further into the nature and extent of this support. What is it for metaphysics to be given such 'support' in this manner? To begin to answer this question we might take our cue from the way in which certain elements of scientific theories are taken to receive support from the empirical success of those theories. So, many realists these days would insist that such support should only be understood as transmitted to those entities, mechanisms or whatever, that play the appropriate explanatory role in accounting for that empirical success. Thus, they argue, we should adopt a realist stance towards electrons because they play the appropriate role in explaining certain electromagnetic phenomena etc. The obvious question, then, is, can we adopt a similar 'explanationist' line and take such support to be similarly transmitted to a metaphysical device like the Principle of Identity of Indiscernibles?

If one were to adopt the same line-namely, that such support should be taken to be transmitted to those mechanisms or devices that play the appropriate explanatory rolethen one must further ask, what role do we take Leibniz's Principle to play in this context? Indeed, what is it that we take it to be explaining? An obvious answer to that last question might be: the Principle metaphysically explains the individuality of the electrons (or whatever) by virtue of 'grounding' (to use a currently popular notion) that individuality in the properties of the electrons. In other words, it functions as a so-called Principle of Individuality and indeed, insofar as one can consider it as enabling us to metaphysically ground and thereby metaphysically explain the particles' identity and individuality, one might even argue that it offers the 'best' such explanation: it allows us to avoid invoking some apparently mysterious 'Lockean substratum', or having to appeal to some primitive notion such as haecceity, or somesuch, and it appeals only to the properties of the particles concerned. Thus one might argue that just as the realist can articulate and defend forms of Inference to the Best Explanation in the context of her analysis of science, so the metaphysician can do likewise, where 'best' is similarly cashed out in terms of "simplicity, comprehensiveness, elegance and so on" (Sider et al. 2007, 7).

With that in hand, one could then articulate an argument to the effect that just as electrons receive appropriate support from the success of the relevant theory by virtue of their featuring in an inference to the best explanation in that context, so PII likewise receives support (from classical physics at least) for analogous reasons. Generalising, the argument can be expressed as follows (taken directly from Saatsi 2017):

MC1 Both metaphysics and science employ inference to the best explanation.

MC2 We have no reason to think that if explanationism is truth-conducive in science,

it is not so in metaphysics.

MC3 We have a positive reason to think that if explanationism is truth- conducive in science, it is also so in metaphysics.

Hence, explanationism in metaphysics is also truth-conducive and, in terms of our discussion here, metaphysical devices that play the appropriate explanatory role may be taken to be supported by the empirical success of the given theory.

However, the argument, as stated, is hugely contentious and the above three assumptions can all be rejected. In particular, Saatsi argues that MC2 is false, since even if explanationism is truth-conducive in science, we actually do have reason to conclude that it is not in metaphysics. First of all, as is well known, when it comes to science, this truthconduciveness must be qualified, since the historical record indicates that the relevant 
explanatory inferences are reliable only in limited respects (Saatsi 2017). Thus, anyone maintaining some kind of methodological commonality between science and metaphysics in this respect is going to have to face up to the crucial difference that there appears to be nothing equivalent that might motivate similar limitations on the metaphysical side. When it comes to realism, of course, these limitations on the reliability of these explanatory inferences are manifested through various forms of 'selective realism', where what is selected may be 'working posits', or 'structure' or whatever. Again, there is nothing to suggest any similar kind of manifestation on the metaphysics side, and indeed it is hard to get a clear picture of what a 'selective' metaphysics would look like.

Furthermore, Saatsi persists, in order to be plausible, forms of explanationism should meet the requirement that they mesh with our understanding of how our cognitive capacities and ways of reasoning are the results of both cultural and natural evolution. So, on the scientific side of things, the discovery of the causal connections that sit at the heart of many scientific achievements is rendered non-mysterious by virtue of the fact that the relevant mode of explanatory practice, namely causal-mechanical, is exemplified in everyday cognitive situations. In part, it is this that underpins the claim that is often made that science is just an extension of the form of reasoning we deploy in 'everyday life'. Again, however, it is not at all clear that something similar can be said with regard to the kind of explanatory practices supposedly exhibited in metaphysics and we don't seem to have the aforementioned 'meshing' (I'll come back to this at the end).

As for MC3, again Saatsi raises doubts as to whether we do in fact have any positive reason along the lines suggested. So, a sympathetic metaphysician might acknowledge that, of course, causal-mechanistic explanations are inappropriate in the metaphysical context but that, nevertheless, there are features of other kinds of explanatory formats that have been put forward in the philosophy of science that have analogues in metaphysics. Two in particular have been emphasised: the power to convey understanding and unificatory power. However, as Saatsi again argues (Saatsi 2017), understanding is a tricky concept even on the scientific side, and even if one can relate the introduction of metaphysics to such understanding (and again I'll come back to this), the claim that it is truth-conducive is highly dubious. As he notes, disputes over whether understanding has been achieved occur not only between different scientific fields, but even within a particular field, where scientists themselves may disagree whether a particular theoretical feature provides understanding or not: since I've already mentioned Leibniz, it is worth noting that he famously rejected Newton's theory of gravity on the grounds that it featured an 'occult' entity and thus did not supply the requisite measure of understanding.

Of course, for Leibniz this understanding could only be provided in what we would now call metaphysical terms and some would argue that it is by underpinning such understanding that metaphysics proves its value. Indeed, it is along these lines that we can draw the distinction between 'shallow' and 'deep' realists (cf. French 2018), with the former eschewing as much metaphysics as they can, and the latter appealing to various metaphysical devices in the way I shall be considering shortly. Returning to the central issue, this point is of no help to the advocate of MC3, of course, since by advocating a particular role for metaphysics, it further illuminates the differences with science.

As for unificatory power, attempts to set this at the heart of scientific explanation are well-known but also, as Saatsi notes, highly contentious. The Friedman/Kitcher programme of explanation-via-unification is widely agreed to have run into the sand. Furthermore, as Saatsi goes on to remark, even if one were to adopt the weaker line that unificatory power counts as an explanatory virtue, the way that this virtue features in 
current well-developed accounts of explanation does not give much in the way of hope to the advocate of MC3, at least not as things stand.

So, according to what has come to be known as the 'Woodwardian' account, explanation involves the exhibiting of a kind of counterfactual dependence that describes how the explanandum would change under various conditions (Woodward 2003). The relevant modal information is then captured via those generalisations that remain invariant under changes in certain variables that, when it comes to scientific explanations anyway, represent certain determinate properties. As Saatsi says, to the extent that it can be related to the degree of invariance of a generalisation, unificatory power can be regarded as an explanatory virtue. But, he goes on, it is one of several that have to be weighed against one another; furthermore, as a virtue, unificatory power attaches to distinct explanations, rather than theories. That one theory might be deemed to have more explanatory power that another by virtue of exhibiting more unificatory power is a derivative result at best and even then, only if the relevant explanations in their different theoretical contexts can be compared along Woodwardian lines. Thus, Saatsi continues, there is little support from the scientific side for the claim that the more unificatory theories are the likeliest to be true, and any attempt to export such developments, or their analogs, into metaphysics is going to have to take account of the relevant contextual complexities and nuances, at the very least.

Indeed, Saatsi's conclusion is that the 'strategy of methodological similarity'-namely the strategy that aims to justify metaphysical explanationism on the basis of explanationism's role and epistemic status in science-comes under severe challenge from observations such as the above. Now, there might be ways for the metaphysician to meet this challenge, of course. Saatsi himself has argued that the above kind of Woodwardian counterfactual approach can be extended from scientific to 'geometrical' explanations (2016). Consider, as a toy example, a straight stick parallel transported over a closed path around a sphere (see https://en.wikipedia.org/wiki/Parallel_transport); when the stick is returned to its starting point, one finds that it has rotated by a certain angle. Saatsi argues that the explanation of this angle is geometrical by virtue of it hinging on the dependence of that angle on certain non-local geometrical features, such as the area inside the loop and the radius of the sphere. Crucially, this explanation is asymmetric in a way that meshes with a Woodwardian 'manipulationist' account in which the relevant counterfactual information is provided: basically, if the area inside the loop or the radius of the sphere were different, then the angle would be different. Saatsi then goes on to extend this approach to kinematic explanations in classical mechanics more generally and it has even been suggested that it can accommodate the explanatory role of symmetry principles in physics (French and Saatsi 2018). The metaphysician might find succour in these developments but if metaphysical explanations are to be accommodated along similar lines, the relevant dependences need to be set out and the appropriate counterfactual information made explicit. If we touch base with our go-to example of the Principle of Identity of Indiscernibles, it is just not clear how that might be done, to say the least.

Alternatively, the metaphysician might argue that metaphysics picks up support from science directly via a form of confirmational holism (this is perhaps what is behind Hawley's view, noted above). Here she might draw on a different kind of analogy, that of the classic indispensability arguments for mathematical realism. However, this road also seems blocked, as I shall now indicate (again, see Saatsi 2017).

So, consider the original Quinean form of these kinds of arguments: we should believe our best scientific theories to be true; if we so believe, then we should believe the ontic commitments of such theories; the ontic commitments of any theory are those objects over which the theory quantifies; our best scientific theories quantify over mathematical objects; 
hence we should believe in the existence of mathematical objects. Now, as Saatsi notes, the idea of taking our 'best' theories as literally true is famously problematic (never mind what is meant by 'best') but a bigger and more obvious obstacle is that metaphysics does not supply the kinds of 'objects' over which appropriately formalised versions of scientific theories could be said to quantify (do first order formulations of classical statistical mechanics quantify over haecceities, for example?!). That whole approach just seems a non-starter.

But perhaps the metaphysician could edge back towards the explanationist camp and point to recent formulations of the so-called 'Enhanced Indispensability Argument' (see, for example, Baker 2009), that run (crudely) as follows: we should believe those ontic commitments of our theories that play an indispensable explanatory role; mathematical entities play such a role; hence we should believe in mathematical entities. This argument has provoked a number of responses but the underlying approach of them all is to accept that mathematics clearly plays some role with regard to the relevant explanations but deny that this role carries ontological commitment. Thus Strevens $(2008,331)$ suggests that mathematics acts as an 'explanatory tool' in the sense that it is through grasping mathematical dependences and independences that we are able to grasp causal dependences and independences. But of course, this is not the same as playing the sort of explanatory role that supports the kinds of existence claims associated with the Enhanced Indispensability Argument. And when it comes to metaphysical entities, it is not at all clear that they can be conceived of as explanatory tools even in this limited sense.

Alternatively, Melia (2000) and Saatsi (2011) have argued that mathematics can be regarded as the 'scaffolding' for our explanations in science, or, more generally, that it plays a representational role, but that, again, this carries no ontological weight. Here the metaphysician might have more room for manoeuvre, as it were: she could argue that in articulating what our theories tell us about the world, metaphysical entities act as a kind of scaffolding that may then be refined or even taken down and reconstructed, as theories develop and change. Consider the objects of classical mechanics, for example. These are, or were, at least initially, conceived of along the lines of 'everyday' objects, albeit subjected to certain idealisations (e.g. point masses etc.), which conception then carried with it, as a kind of scaffolding, certain metaphysical devices regarding composition, identity (e.g. the PII) and so forth. Thus such devices may be seen as playing, or contributing to, a broadly representational role, in helping to spell out how the world is, according to the theory. I'll touch on this again in the context of realism below but given this, we might think of metaphysics receiving some support, albeit attenuated, from our successful theories.

Even granted all that, however, it is worth noting that any such support-as given by classical physics, say, to the Principle of Identity of Indiscernibles-is not unequivocal, as a result of the underdetermination of the metaphysics by the relevant physics. So, we just noted that on some understanding of metaphysical explanation, PII could be regarded as the 'best' explanation of the individuality of the particles. But if we were minded to, we could eschew the Principle, perhaps because we want to insist on the Scholastic distinction between individuality and distinguishability, and thus maintain that the Principle can only explain the latter, not the former, which hence requires a different ground, such as haecceities or 'primitive thisness'. Following this line, the latter notion would be the relevant 'best' explanation and of course this is also supported by classical physics (French and Krause 2006, ch. 2). The metaphysics - of individuality in this case-is thus underdetermined by the physics. And we can continue: take the notion of 'property' 
appealed to in the expression of PII-is this to be understood as an instantiated universal or a particular, that is, as a trope? Again, the physics offers no support.

As a result of this kind of underdetermination, the claim that metaphysics draws positive support from modern science once more needs to be considered with some care. After all, in the case of two scientific theories that are empirically equivalent and hence underdetermined, we would be tempted to conclude that neither can be taken to be supported over the other. Of course, cases for which no possible evidence could be obtained that might differentially bear on the theories are notoriously hard to establish in science, with the bestknown examples being drawn from space-time physics. The classic (in both senses) example is that of the underdetermination of claims regarding the velocity of the material universe in Newtonian physics. Others can be found in the modern context, where different models of General Relativity can be constructed between which no possible observation could distinguish.

In these kinds of cases, the usual response is to argue that such claims, or models, can in effect be hived off from the theory in general, thus inoculating the latter from the underdetermination and effectively allowing it to retain empirical support. Obviously such a manoeuvre is not available in the metaphysics cases, given the centrality of the relevant claims for the respective positions-in effect, when it comes to metaphysics we are not so easily able to distinguish something similar to a 'model' from that which is playing the part of the 'theory'. One might be tempted to broaden the position concerned in order to be able to make something like the above distinction and say something along the lines of the following: whether we cash it out in terms of haecceities or the Principle of Identity of Indiscernibles, the idea that the objects of physics are individuals is supported by classical physics. In that way, the specific metaphysical claims may be hived off and the underdetermination deflated. Unfortunately, this leaves the metaphysical 'theory' that is not underdetermined pretty broad and bland, to the point where it is close to empty. There is precious little metaphysical flesh on the bones of the claim that 'particles are individuals'. Close to empty but not entirely so, since when we move to the quantum context, of course, we find a further form of underdetermination between that claim and the alternative, 'particles are non-individuals'. I'll come back to that shortly.

Given the underdetermination of the Principle of Identity of Indiscernibles and the notion of haecceity by the relevant physics, perhaps we should be wary of asserting that these metaphysical elements draw support from that physics, even in an attenuated sense whereby such support is somehow secondary to or parasitic upon the kind of support that empirical results give to theories. Perhaps the best we can hope for is some sense of these elements being compatible with or meshing with that physics. Of course, that still leaves a dilemma for the realist, if she feels her realism requires filling in with metaphysical 'content' in the sense of incorporating one or other of these metaphysical elements; but that's a big 'if' of course (French 2018).

More generally, given this underdetermination, one might wonder what the critics of 'mainstream' metaphysics and others are hoping for when it comes to the engagement between metaphysics and science. Leaving aside the sociological concern that more metaphysicians should understand modern science, it cannot be a matter of insisting that metaphysicians should come up with views that are supported by physics, in the usual sense of support. In terms of that sense and given the above underdetermination, one could conclude from the above that metaphysics will always float free of science. But perhaps the real concern is that metaphysicians should not continue to develop, deploy and generally utilise metaphysical elements that have been ruled out by science, so let me now turn to the negative impact of the implications of physics. 
Again, let me take the above and much used example of the Principle of Identity of Indiscernibles. Here the impact of quantum physics is well known: if we take quantum objects to be individuals, then, assuming the standard formulation of quantum mechanics, it can be argued that consideration of their aggregate behaviour, (whether as bosons or fermions, as described by Bose-Einstein and Fermi-Dirac statistics, respectively), implies that objects of the same kind possess all properties in common and hence that Leibniz's Principle is violated (French and Redhead 1988). In that case, some other grounding for individuality must be chosen, such as haecceities again, or so it might seem (French and Krause 2006). Alternatively, we may follow the lead of the early quantum physicists and take this aggregate behaviour as indicating that such objects should not be regarded as individuals in the first place, in which case the Principle is effectively obviated (and forms of non-standard set theory may be introduced to help formally capture the sense of nonindividuality involved; again see French and Krause 2006, ch. 7). Thus, we appear to have a case of physics having a negative impact, whereby it rules out or, perhaps we might say, falsifies (at least in a loose sense) the relevant metaphysics.

But again, this is too quick. Such, at least, was the story until fairly recently. Drawing on previously little considered work of Quine's, Saunders has effectively modified PII so as to allow certain kinds of relations to play a distinguishing role. In particular, symmetric but irreflexive relations of the form '... has opposite [property] to ...' ground a kind of 'weak' discernibility. Saunders has then argued that two fermions can exist in certain states in which they can be described as each entering into the symmetric and irreflexive relation of '... has opposite spin to...' Thus, for fermions (such as electrons, for example) at least, a form of 'weak' discernibility can be established, via this modified form of Leibniz's Principle, which can then be used to ground their individuality. Whether this result can be extended to bosons remains contentious (Saunders 2003; Muller and Saunders 2008; Hawley 2009; Huggett and Norton 2014), as is the appeal to relations holding between objects in order to ground the individuality of those objects (since there's the obvious concern about possible circularity). Nevertheless, the point is that here we appear to have an example of the 'regeneration' of a piece of metaphysics, in the sense that a metaphysical device (in this case Leibniz's principle), that was previously rejected, given the impact of physics, is shown to be compatible once more with that physics. Of course, this has been achieved not by undermining the previous negative impact, but by suitably adjusting or revising the metaphysical device concerned. Indeed, it has been argued that the revision in this case is such that we should not regard the device as a variant on Leibniz's Principle at all (Ladyman and Bigaj 2010) but we can perhaps be charitable and think of this shift in terms of an analogy to Lakatos' methodology of scientific research programmes (French and McKenzie 2015). Of course, the analogy is not exact, not least because it is unclear what would count as the 'hard core', or 'positive heuristic' etc. in such cases, but we can at least conclude that the ruling out by physics of a particular piece of metaphysics may not be definitive, since the latter may be rendered compatible once more, albeit in a new guise.

More importantly, perhaps, for current purposes at least, this regeneration was achieved by drawing on a new (in this context) piece of metaphysics developed by Quine (1976), namely weak discernibility. Now, of course, Quine himself espoused a form of naturalism and, indeed, he took the impact of quantum physics seriously, arguing that the aforementioned view that quantum particles are non-individuals in some sense motivates their elimination as fundamental elements of our ontology in favour of space-time points. And indeed, in order to articulate the differences between stronger and weaker senses of discriminability, Quine uses the ordinal numbers as examples. Nevertheless, the general context is that of formally articulating the various grades of discriminability of objects in a 
given interpreted formal language and we can take Saunders' move as exemplifying point (2) in our initial core claim, namely that elements of metaphysics-such as weak discernibility — which are apparently developed without close regard to any relevant science, may in fact be deployed as tools to help articulate a particular interpretation of quantum mechanics (namely one in which the relevant entities are individuals, subject to a form of the Principle of Identity of Indiscernibles).

\section{The Metaphysical Toolbox}

Here's another example of a metaphysical programme that appears to have gone through similar phases of being supported by, or at least compatible with, the relevant science, then ruled out or deemed incompatible, before later developments emerge suggesting a possible 'regeneration', thus again supporting (2) above: dispositionalism. Originally articulated in the context of 'everyday' objects such as vases and windows, with dispositional properties such as 'fragility' etc. taken to be reducible to categorical properties, it has subsequently been extended to the latter, so that supposedly fundamental properties such as charge have come to be understood as themselves dispositional in nature. Here's one very well-known expression of this view:

Physics tells us what result is apt to be produced by the having of gravitational pull or of electromagnetic charge. It does not tell us anything else about these properties. In the Standard Model the fundamental physical magnitudes are represented as ones whose whole nature is exhausted by their dispositionality: that is, only their dispositionality enters into their definition. Properties of elementary particles are not given to us in experience: they have no accessible qualitative aspect or feature. There is no 'impression corresponding to the idea' here. What these properties are is exhausted by what they have a potential for doing, both when they are doing it and when they are not (Molnar 1999, 13).

This dispositionality is typically characterised in terms of the so-called 'Stimulus and Manifestation' characterisation:

$$
\forall x((\mathrm{P} x \& \mathrm{~S} x) \rightarrow \mathrm{M} x)
$$

where $\mathrm{P}$ is the relevant property, such as charge, $\mathrm{S}$ the stimulus and $\mathrm{M}$ the manifestation. Thus, taking charge as our example, we can imagine a situation, a possible world perhaps, whereby we have a sole object - an elementary particle, say - that is charged (and the fact that we can imagine such a situation in which we have such a lonely object is typically taken as underpinning the view that charge is an intrinsic dispositional property; I'll return to this feature shortly) and when we bring up another charged object, the two experience a force, attractive or repulsive, depending on the signs of the respective charges. The bringing up of the second charge counts as the stimulus and the force experienced (or, some would insist, the acceleration that results) is the relevant manifestation. All there is to charge, it is claimed, is captured by this characterisation (in particular, the idea that there is some quiddity that makes charge charge, is denied).

On this basis, or so it is claimed, one can obtain the corresponding laws (see Bird 2007; for criticism see Vetter 2009) and their necessity is dispositionally underpinned: in any possible world that contains objects with property $\mathrm{P}$, the presence of the stimulus will yield the relevant manifestation; hence in those worlds, the corresponding laws will hold. 
Indeed, with the decline of the Armstrong-Dretske-Tooley account of that necessity as a manifestation of necessary relations between universals, the dispositional account has come to be seen as the main contender for a modal account of laws, in opposition to the Humean/Lewisian view which eschews any such necessity in the world and takes laws to be those descriptions of regularities that happen to feature in our best theoretical systems (however 'best' is determined). This role that dispositionalism has acquired is crucially supported by claims, such as the above, that it finds support in modern physics (where 'support', again, has to be understood rather broadly).

However, such claims have been challenged and we can view such challenges as examples of the negative impact of science in which the relevant metaphysics is effectively ruled out. Thus, note that the above quote refers, as support, to the Standard Model of current physics. This is the model that, famously, encompasses the electromagnetic, the weak nuclear and the strong nuclear interactions and classifies all known elementary particles. It incorporates two features relevant for our discussion here: one is the so-called Higgs (or perhaps better, given all those involved in its postulation, the 'ABEGHHK'tH') mechanism via which mass is acquired via coupling to the Higgs field. The recent discovery of the Higgs boson - the quantum excitation of this field-has been taken to provide significant support (in the stricter sense) for the Standard Model. The other has to do with certain kinds of symmetries, namely the global Poincaré symmetry that all relativistic quantum field theories incorporate and the local $\mathrm{SU}(3) \times \mathrm{SU}(2) \times \mathrm{U}(1)$ gauge symmetry that effectively defines the Standard Model and covers the three fundamental interactions (nuclear, weak and electromagnetic, respectively). Neither of these crucial features of the Standard Model can be captured by dispositionalism (at least not straightforwardly).

Thus Bauer argues, first, that the grounding of mass in the Higgs field means that it cannot be regarded as an intrinsic property (Bauer 2011). This in itself sets a challenge for the advocates of dispositionalism who typically take the fundamental properties of physics, such as mass, charge and spin, to be not only dispositional, but also intrinsic in the sense that they are not grounded in any other properties. The confirmation of the existence of the Higgs mechanism implies that mass must be regarded as extrinsically grounded-namely, grounded in the Higgs field (Bauer 2011). Furthermore, insofar as this field extends throughout the universe, mass must be regarded as 'ultra-grounded' in the sense that it is grounded in the whole physical environment in which the relevant particle finds itself (Bauer 2011, 97-99). At the very least this complicates the picture underlying the 'Stimulus and Manifestation' mechanism: in the case of mass, we cannot, strictly, imagine a situation in which there is a lonely object possessing mass, since for there to be mass, there must be the Higgs field. But there are, of course, avenues by which the dispositionalist can respond to this concern and certainly, she may insist that even if mass must be regarded as extrinsic (and that itself may be accommodated within dispositionalism, if we accept a relational or 'global' form of this view that allows dispositional properties to be non-monadic or non-local), charge and spin need not be (although in the case of spin, she may not have good cause to be so sanguine, given one reading of the Spin-Statistics Theorem; see French forthcoming).

However, a more significant concern for the dispositionalist is that of accounting for the symmetries of the Standard Model. Their heuristic role is well documented and the likes of Wigner have famously insisted that they should be regarded as 'meta-laws' that constrain the dynamical laws that philosophers have tended to focus on. Thus, in the context of the above considerations of particle identity and individuality, consider the symmetry underlying such considerations when it comes to quantum mechanics, namely Permutation Symmetry: putting things crudely, this imposes invariance under permutations of the 
particles and the relevant constraint manifests itself in the requirement that the Hamiltonian must commute with the particle permutation operator. As a result, the relevant Hilbert space is divided into non-commuting sectors, one of which corresponds to Fermi-Dirac statistics (obeyed by electrons, for example), another to Bose-Einstein statistics (obeyed by photons), others (if we have more than two particles in the given assembly) corresponding to so-called 'para-statistics' (which quarks were at one time thought to obey).

Or consider the symmetries of the underlying space-time of the Standard Model, namely Minkowski space-time, which are captured by the Poincaré group, the representations of which yield a classification of all elementary particles in terms of their properties such as mass and spin. Or, again, the 'internal' gauge symmetries of the Model, which yield both the well-known property of charge, in the case of electromagnetism, as well as the more 'exotic' ones such as isospin and colour in the cases of the weak and strong nuclear forces, respectively.

The question, then, is how is the dispositionalist to accommodate these features of the Standard Model? Certainly the standard 'Stimulus and Manifestation' framework, as sketched above, appears unable to accommodate them and other obvious manoeuvres by the dispositionalist also run into difficulties (see French forthcoming). ${ }^{1}$ Thus, for example, she cannot simply insist that fundamental dispositional properties are correlated with these symmetries $^{2}$ since such correlation, alone, cannot accommodate the role of symmetries in constraining the relevant laws. Consider: on the basis of the standard view of dispositions, incorporating the 'Stimulus and Manifestation' characterisation, it can be shown that the relevant laws 'flow from' or are supervenient upon certain dispositions. Now of course, one could drop the constraining role of symmetries - which would of course require giving some account of how they appear to play such a role in modern physics — and demonstrate that they too flow from or are supervenient upon the relevant dispositional properties, via the laws. But so far as I know, noone has ever done this and in the absence of such a demonstration (and I don't know how one would even begin) such a suggestion must remain entirely speculative.

Furthermore, and returning to the central theme, in this case at least, we have the advantage that the dispositionalist, through the work of Bird and others as indicated above, presents us with a clearly articulated account of how that framework yields scientific laws. Thus we don't need to worry about the nature of metaphysical explanation or such matters, in these cases, since all we need to show is that this account cannot accommodate the above symmetries (French forthcoming).

The upshot then is that here we have further examples of the negative impact of science upon metaphysics. Perhaps it is too strong to say that the metaphysics has been 'falsified' in these cases but certainly one could contend that dispositionalism, as standardly understood (that is, in terms of the 'Stimulus and Manifestation' characterisation) has been ruled out in the context of modern physics.

Now, as it turns out, that does not hand physics on a plate to the Humean, since the latter's view of laws also faces certain difficulties in accommodating such symmetries and indeed the Standard Model in general (French forthcoming). Again, an immediate question is, how are we to accommodate the constraining role of symmetries within such a view?

\footnotetext{
${ }^{1}$ Of course, as one of the referees pointed out, one might raise an eyebrow over the assumption that the Stimulus and Manifestation characterisation can even accommodate fundamental laws, given the claim that the fundamental forces involved need no stimulus to be effective. However, the dispositionalist may respond that this amounts to question begging and for myself, I'm willing to be charitable and grant their account of laws since even if this is accepted, it is difficult to see how symmetries are then obtained.

${ }^{2}$ I am grateful to one of the referees for suggesting this.
} 
One option would be to reject such a role, just as Humeans reject the supposed 'governing' role of laws (see, for example, Beebee 2000) and argue that the appearance of constraint is nothing more than a manifestation of the way symmetries function heuristically in theory discovery and pursuit. Nevertheless, the details need to be worked out. Consider, the Humean view has two core features: a metaphysical claim to the effect that reality consists of a 'mosaic' of particular events, distributed over space-time and between which there are no modal connections, or indeed, relations of any kind except spatio-temporal ones; and an epistemic claim to the effect that of the various regularities that we discover in this mosaic, the ones we deem to be laws are the ones we can accommodate within our 'best' theoretical system, where 'best' here involves some balancing of simplicity with 'strength' (typically cashed out in terms of explanatory power). When it comes to the mosaic, the Humean could suggest that symmetries feature as 'meta-' regularities that hold across the regularities that are deemed to be laws. And that these will then likewise be represented within our 'best' systems, just as those we deem to be laws are.

But obvious concerns arise: first, how will this notion of 'best' be cashed out when it comes to symmetries? We can more or less grasp how different laws may differ in their explanatory power, but there is still considerable work to be done in explicating how symmetries function in explanations (see Lange 2012). More fundamentally, how could the way in which such symmetries hold across laws, qua regularities, in the mosaic be reflected in the relationship between their representational counterparts (that is, the law-statements and symmetry-statements) within the 'best' system formalisation? This is not clear and the worry is that the Humean may have to face a similar kind of tension that Hall identifies in accommodating the distinction between laws and initial conditions that we find in scientific practice in both the picture of the mosaic and the best system (Hall 2015).

An alternative option, then, would be to effectively rummage in the metaphysical toolbox again and deploy some other device to capture the metaphysical nature of these symmetries. One such is Vetter's recent account which effectively replaces the standard understanding if dispositionalism with one based on the notion of 'potentiality' (Vetter 2015). Here the core idea is to drop the 'stimulus' component from the 'Stimulus and Manifestation' characterisation and capture the requisite sense of modality via the simple prescription ' $x$ can $M$ ', where $M$ is the manifestation. Thus to say that a vase is fragile is to be captured via the claim 'the vase can shatter' which expresses the fact that the vase has the potential to shatter. Furthermore, the relevant possibility here both depends on context and is gradable. Thus, for example, in the context delineated, in part, by my kitchen floor, the vase has a greater potential to shatter than my dinner plate (made as it is of quite tough pottery!). One can see the contrast with dispositionalism as follows: the dispositionalist will accommodate this context dependence in terms of a property that comes in degrees, some of which will be sufficient for the correct application of 'is fragile'. On Vetter's account, by contrast, being fragile is a matter of having the right potentiality to a contextually sufficient degree (Vetter 2015, pp. 21-22).

The suggestion then is that we treat this account as a metaphysical tool and apply it, not to objects, but to symmetry principles themselves (where this may then require us to reinterpret the relevant sense of 'potentiality', of course ${ }^{3}$ ). Thus, if we take ' $x$ ', in the above formulation of Vetter's account, to be the given symmetry we can accommodate the

\footnotetext{
3 And one way of understanding it is in terms of the possibilities offered by the mathematics in terms of which the symmetry is represented. There is more to say here but in those terms it doesn't seem that there is much difference in saying that a vase has the potential to shatter and a symmetry has the potential to yield various properties.
} 
relationship between that symmetry and certain fundamental properties or kinds in physics. Consider the way that spin, for example, 'drops out' of the Poincaré symmetry by virtue of this property functioning as one of the indices of the (positive energy, unitary, irreducible) representations of the group (the other index being mass). If we stretch the notion of manifestation from something that is typically regarded as observable to a property such as spin (which of course has observable consequences), we could take the symmetry as having the potential to yield the property in the right context - that being relativistic spacetime-and spin could be regarded as the relevant manifestation in this case. Likewise, if we took the $x$ in the above formulation to be Permutation Symmetry, we can understand the properties of being bosons or fermions as the Ms. Furthermore, Vetter's distinction between potentiality and 'mere' possibility in terms of the "localized counterpart of a nonlocal modality" (Vetter 2015, 23), can then be cashed out in the latter case in terms of Permutation Symmetry yielding a whole range of possible particle statistics (the aforementioned parastatistics), only two of which (the Bose-Einstein and the Fermi-Dirac) appear to be manifested in the actual world.

Of course there are concerns one can raise about this. First, Vetter motivates her account by considering the way we use certain phrases in ordinary language as applied to 'everyday' situations. Thus her account is based on considerations far removed from those associated with physical symmetries. But of course one could say the same about Quine's notion of 'weak discernibility' as subsequently applied to physics by Saunders. Indeed, this exemplifies my central claim: metaphysics may still be useful to the philosopher of physics even if it is grounded in considerations that appear to have nothing to do with modern physics. And in Vetter's case, by deploying her notion of 'potentiality' in this context, we may 'regenerate' the dispositionalist programme, in the quasi-Lakatosian sense indicated above.

Secondly, one might wonder whether a symmetry can legitimately sit in the place of the ' $x$ ' in Vetter's characterisation (see French forthcoming). But first, there are other tools that are available and secondly, if one sees these, and Vetter's account, as modifications of dispositionalism, then as in the case of weak discernibility and the Principle of Identity of Indiscernibles, one can draw the analogy again with Lakatos' Methodology of Scientific Research Programmes: we keep the 'hard core' represented by, in Vetter's account, the potentiality for a property to manifest in certain ways, and we drop 'auxiliary' elements of the 'periphery', such as the stimulus requirement. Of course we should not push this analogy too far but we can appreciate, at least, how talk of dispositionalism being 'falsified' or irrevocably ruled out of court, is perhaps too strong.

In summary, then, what I am suggesting, following the line laid down in French and McKenzie (2012) is that although modern physics has a major negative impact on certain devices that feature prominently in 'mainstream' metaphysics, there are other devices that the philosopher of science can utilise as tools in articulating the relevant metaphysical nature of modern physics and, further, insofar as those latter devices or tools bear some kind of family resemblance to the former, we can bring the above two claims together and see this process of ruling out and further tool deployment as analogous to Lakatos' programme more generally. 


\section{Worries}

There are a number of worries that one might have about this picture. Here I shall consider three.

First of all, one might question whether 'mainstream' metaphysics, however that is to be delineated, really does 'float free' from science or at least, if not entirely then sufficiently so for it to become such a target for opprobrium.

And this can be taken to crystallise in the further question, to what extent can these metaphysical tools taken from the mainstream 'toolbox' actually be scientifically disinterested? Consider again the notion of weak discernibility, taken by Saunders from Quine's contributions to the toolbox: Quine himself was certainly not disinterested in science and although the notion of weak discernibility was clearly not motivated specifically by considerations of physics, quantum or classical, one could perhaps argue that the whole framework of his discussion of discernibility and identity may have been influenced by his reflections on science, even if only indirectly. Likewise, although Vetter's extension of dispositionalism does not draw on science-based considerations, but rather on those to do with our use of language, one could also argue for an indirect connection via that use.

But if that seems too tenuous, and the worry correspondingly rather weak, one must surely admit that even though some metaphysicians like to portray themselves as exploring the far reaches of metaphysical 'space', devices such as weak discernibility and potentiality seem to emerge from possible worlds very close to, if not identical to, this one! And given not only reflections upon language use and the like but also the role of intuitions in conceiving of such possibilities, and the way in which such intuitions may be laden with some theory or other, even if only along the lines of 'folk psychology', perhaps one could still maintain that few if any mainstream metaphysical tools are entirely scientifically disinterested.

Indeed, suppose one were to agree with Lowe that "[m]etaphysics deals in possibilities" (Lowe 1998, 11) one might still insist that such possibility is grounded in conceivability and that the latter is bounded and grounded in certain ways. Here one might ask the question: what are the general conditions governing conceivability or imaginability, such that conceiving or imagining a world or situation is deemed sufficient to provide evidence for the possibility of such a situation? One obvious answer is that there are no such conditions or constraints, so that the mere act of conceiving or imagining a situation is sufficient for it to be deemed to be a legitimate possibility. Following this line, one would have to conclude that conceivability is not defeasible, in the sense that there is no consideration that would undermine the relevant evidential link between conceivability and possibility (Yablo 1993; Chalmers 2002).

For many people, of course, this is too strong and the discovery that one's conception is inconsistent would be one such consideration, undermining the link between that conception and a 'genuine' possibility. Now of course, that discovery may not be straightforward and so certain conceivable situations may come to be removed from the space of possibility as they are further investigated via logical analysis, for example. A more interesting and contentious example would be Hacking's critique of Black's 'two-spheres' dismissal of our old friend the Principle of Identity of Indiscernibles. In brief, the dialectic is as follows: Leibniz's Principle can be taken as stating that one cannot have two things that are (absolutely) indiscernible. Black argued that the Principle cannot be necessary since one could conceive of a world in which there are two iron globes, one mile apart, say, that are indiscernible (Black 1952). Hence the Principle is only contingent (see, again, 
Casullo 1984; and then French and Redhead argued that as such it is false, Saunders effectively resuscitated it and so the story continues...). Hacking insisted that 'mere' conceivability is not enough and for such a conception to count as a genuine possibility it must incorporate an appropriate spatio-temporal background (in terms of which, at the very least, one can say that the spheres are one mile apart; Hacking 1975). But once that is admitted, the defender of the Principle can effectively neutralise Black's argument by, for example, choosing a relativistic space-time in terms of which the two-spheres situation can be transformed into one-sphere and a highly curved background space-time, thereby redescribing the apparent counter-example in a way that removes its force against the Principle (in other words, there would be a kind of underdetermination between the 'appearances', in some sense, and the relevant description in terms of spheres and spacetime). There is much that one could say about this manoeuvre (for an example, see French 1995) but the point is that as far as Hacking is concerned, conceivability has to be constrained via the incorporation of an appropriate spatio-temporal background in order for it to be evidence for the relevant possibility.

Thus, we can conceive (ha!) of a kind of 'conceivability spectrum', ranging from unconstrained conceivability, which should perhaps come with a metaphysical 'health warning' as it may include Meinongian objects and inconsistencies in general, to logically constrained conceivability, with the Principle of Non-Contradiction, of course, acting as a significant constraint, to conceivability constrained by intuitions, with regard to which we can draw on recent discussions of the role of such intuitions in philosophy (see, for example, Pust 2017), to metaphysically constrained conceivability, which may come before the previous entry depending on what the relevant metaphysical and 'intuitive' constraints are, ${ }^{4}$ to, finally, physically constrained conceivability (or 'naturalistic' conceivability, perhaps), within which we might distinguish constraints based on classical physics, quantum physics and so forth.

This then generates a corresponding spectrum of views on the defeasibility of conceivability, moving from perhaps the broadest view which is that conceivability is simply indefeasible, so that in effect, anything can happen in the space of possibility (!), to the more restricted but still quite broad view that conceivability is not defeasible by science, or any features of 'the actual', depending on how intuitions are shaped by the actual, to the narrow view that conceivability is defeasible by science, so that only those conceptions that are compatible with current science may be taken to underpin the relevant 'genuine' possibilities. Of course, on this last view, what counts as a genuine possibility is an entirely fallible matter, shifting as science progresses, with a kind of ideal conceivability reached via compatibility with the ultimate 'Theory of Everything'.

An obvious worry about this last case is that it reduces the possible to the actual, since if we have reached the ultimate ToE, there would be no further legitimate possibilities to consider (see, for example, Bird 2014), but as we move between these views we slide from a non-naturalistic stance to one that we might describe as 'naturalistically tinged' to fullon, and perhaps unpalatable, naturalism.

In terms of this spectrum of views, we can respond to the kind of attitude evinced by Lowe and others - to the effect that metaphysicians should not worry too much about the

\footnotetext{
${ }^{4}$ It is worth noting, perhaps, that the relationship between intuitions and philosophical theories or generalisations is typically taken to mirror that between science and metaphysics, in that if the theory or generalisation conflicts with the intuition then this is treated as (defeasible) evidence that undermines said theory or generalisation (Pust 2017), and, conversely if the theory/generalisation has consequences that agree with the intuition concerned then this is taken as support for that theory/generalisation.
} 
impact of modern physics, since metaphysics has to do with the possible, rather than the actual-by acknowledging that the kinds of metaphysical resources or tools indicated above may be generated all along that spectrum. Thus we should perhaps index modal claims to a particular possible world within a particular framework of possibility (or a particular spot on the spectrum of conceivability) and so it is not enough to simply respond to the kinds of concerns that Ladyman and Ross and others have raised by insisting that metaphysics deals in possibilities-where one sits in the spectrum needs to be specified. As a result, the 'space' of possibility becomes multi-dimensional, in a sense, and our metaphysical 'toolbox' is effectively enlarged along these multiple dimensions. But of course, it might be expected that we would rummage around in those volumes of metaphysical space that lie closer to home, on the obvious grounds that the closer we are to 'the actual', the more likely we are to find devices that will be applicable. Indeed, we might even think of 'tagging' the tools we find in this expanded toolbox with an explicit admission of where they lie on the conceivability-possibility spectrum.

In effect, then, I am acknowledging that the initial characterisation of still potentially useful metaphysics as 'scientifically disinterested' was rather crude. There will be degrees of disinterestedness depending on where the metaphysics lies, as it were, along the above spectrum. Thus we should not be surprised if we were to conclude that Quine's 'weak discernibility', for example, is not entirely scientifically disinterested. Vetter's notion of 'potentiality' offers some further complexity, since although it is explicitly scientifically disinterested in one sense, insofar as she notes that it is not compatible with classical physics (by virtue of dropping the stimulus aspect), it is still grounded in 'the actual' in another sense, given the motivation in how we express possibilities in everyday language. Of course, one could adopt a 'hard' approach along eliminativist lines and maintain that only fundamental physics constitutes 'the actual', so that this device of potentiality is being drawn from a domain of possibility relative to that fundamental level. Or one could adopt what to many will be the more reasonable approach that such metaphysical tools may be obtained not just from such parts of 'possibility space' but also from areas or levels of the actual that might be described as 'derivative' or 'emergent' or otherwise non-fundamental as compared to that of modern physics.

The core, 'take home' point is: we may appropriate various metaphysical devices or tools from a wide range of situations, some of them possible, some of them actual, albeit non-fundamental, and put them to use in articulating a kind of 'off-the-peg' metaphysics of science. The alternative is for philosophers of physics to go 'bespoke' and tailor their own metaphysical suit of clothes for whatever theory they happen to be concerned with. Insofar as the aim of the whole exercise is to provide some measure of understanding how the world could be the way modern physics says it is, the latter option faces the obvious danger of obscurantism and, generally, failing to meet that aim by virtue of deploying notions and devices that we cannot readily grasp. I'll come back to this.

However, there is a further related worry that must now be addressed: insofar as these metaphysical tools may derive from thinking about possible worlds different from the actual one-indeed, even far away in 'possibility space' — or from thinking about levels or aspects of the actual far removed from that of modern physics, their usefulness lies in their specific re-casting in terms fit for the latter domain of this world. And that re-casting in order for such devices to be applicable to physics may then effectively 'gut' the device of much of its metaphysical content, leaving merely a kind of shell.

Take the particular device, or set of devices that fall under the term 'dependence': ontic structural realists have argued that this tool can be utilised to help explicate the relationship between physical symmetries, such as that represented by the Poincaré group above, and 
properties of the particles, such as mass and spin; the latter are said to be dependent upon the former (French 2014; see McKenzie 2014 for criticism). But then all the work in this explication seems to be done by the relationship between the group structure, in terms of which the symmetry is captured and the various representations of that group, which yield the properties. The metaphysics seems to merely provide a name for that relationship and little more.

One response would be to insist that that is enough, since even if metaphysical notions can only play this kind of 'umbrella' role, this is still useful, not least in relating a range of such relationships, in this case, and thereby contributing to that aim of achieving some level of understanding (Chakravartty 2013). Thus consider again Vetter's device: in the language of physics we do not have adjectives such as 'fragile' which, Vetter argues, express the relevant sense of potentiality at the everyday level. But we do have the mathematics of group theory, for example, which is the language in terms of which the relevant symmetries are expressed. And we can take this as revealing or encoding the relevant possibilities; so, to consider Permutation Symmetry again: this expressed a whole range of possible particle statistics going beyond that of Fermi-Dirac and Bose-Einstein to include the kinds of parastatistics originally deployed to accommodate quarks, as well as many others. Here one might agree that the content of the possibilities is cashed out by the relevant physics (or rather, mathematics) but nevertheless, what the deployment of Vetter's potentiality allows us to do is to conceive of this content in terms of the potentiality of the symmetry - as a real feature of the world - and to relate that content to other forms of potentiality, including those exhibited by fragile vases at the level of 'the everyday'. Via that last connection, in particular, we may claim to achieve a measure of understanding.

Finally, there is the worry that if any metaphysics does prove useful as a 'tool', philosophers of science then do not have any grounds for castigating, admonishing or otherwise wagging their fingers at metaphysicians who appeal to intuitions or classical or even Aristotelian physics or who ignore or pay little attention to science in general. After all, if 'scientifically disinterested' metaphysics can still be used, why should metaphysicians be at all bothered by their failure to engage with modern physics?! This has been claimed to lead to a form of tension between our initial two claims, as given above (see French and McKenzie 2015). But of course, the point is that metaphysicians were only castigated by the likes of Callender and Ladyman and Ross in the first place because of their claims regarding the implications of their metaphysics for this, the actual world. As long as they refrain from such claims and fully embrace the Lowe line that they are effectively exploring the vast space of possibility, then the tension dissipates.

In effect the role of metaphysics as a tool is doubly conditional (French and McKenzie 2015): it is conditional upon philosophers of physics and naturalistic metaphysicians continuing to take metaphysics down 'off the shelf' and applying it to modern science, instead of making new metaphysical devices to order by themselves; and it is conditional upon those tools and devices actually turning out to be relevant and useful to the interpretation of science as it evolves. This in turn will be dependent on developments in both science and the philosophy of science.

But let us look at those two conditionals a little more closely. What would be involved in constructing such 'new' metaphysical devices? There is an obvious and immediate issue of language-I can introduce whatever new terms I like but if they're not relatable to familiar ones, I'm likely to receive a stare of incomprehension if not incredulity! And if they are so relatable, then any comprehension that follows will of course derive from that attached to whatever current metaphysical device the familiar term designates. Indeed, this is what has happened in the past, even when metaphysics has been explicitly eschewed: 
take Eddington, for example, who famously urged philosophers to stay out of quantum mechanics while it was 'under construction' but then when it came to describing the kind of structural quality that the newly introduced property of spin had, fell back on phrases such as 'patterns of interweaving' (Eddington 1941; cf. Ladyman and Ross 2007). The aim, of course, is to convey some understanding of what the world is like according to modern physics, but, again, for that conveyance to work, it needs to proceed from some already graspable concept that will already be loaded with prior metaphysics (and again we might recall Saatsi's point that when it comes to explanation, our metaphysical devices must be constrained by the requirement of meshing with our understanding of how our capacities and ways of reasoning are the results of cultural and natural evolution).

Now it may be that any such attempt simply fails, as physics advances. So, for example, we can imagine quantum gravity developing in such a way that we end up concluding that it has outstripped our ability to describe the various relations involved or more generally accommodate it using any currently available metaphysical tools, even if scavenged from far off shores of possibility. In that case we may have no choice but to try and come up with our own custom-made devices but given the failures of such attempts in the past-see for example, Eddington (1946) (given that this work remains incomprehensible to most commentators, this is a cautionary tale if ever there was one!)-the prospects do not look good. One will either end up simply reciting the relevant physics, in the absence of any such devices, or effectively having to introduce an entirely new language to match one's new metaphysics. Perhaps one or other of those options is inevitable but let us first at least try exploring what the metaphysics we have to hand has to offer.

And, of course, even with metaphysics understood as a tool in this manner, metaphysicians may not be completely absolved of responsibility to engage with science. After all, we recall that the slogan 'Metaphysics is the science of the possible' puts modality at heart of metaphysics, but as already noted, theories of modality may be effectively ruled out by the physics of the day. Of course, this is mitigated somewhat by the analogy with Lakatosian research programmes mentioned previously: if the dispositionalist programme can be seen as 'progressive' by virtue of Vetter's advance then of course it can't be dismissed as 'falsified' by science. But still, in acknowledging this, one has to accept the impact of physics.

\section{Conclusion}

What I hope to have indicated here is that the relationship between metaphysics and science is more complex and more nuanced than has sometimes been presented. Even if we can make a clear distinction between 'mainstream' or non-naturalistic metaphysics and that which has engaged with science more explicitly, and can come up with examples where principles and positions presented by the former have apparently been undermined by advances in the latter, these principles and positions may gain new 'life' through further developments, suggesting that the appropriate 'unit of interest' in such cases should be metaphysical programmes, however delineated. Furthermore, it has been argued that even in those cases where such positions and principles might be regarded as 'scientifically disinterested', they can still function as tools or devices to be appropriated by the naturalistically inclined philosopher. Such tools may of course be acquired in various ways from all sorts of regions of the 'space' of possibilities or even non-scientific levels of the actual. And in applying them to the theories and models of modern physics, the relevant 
content of these devices must be articulated in terms of that physics-else how could the tools work in the first place?-but that does not reduce such devices to re-descriptions of the physics. Even if only thus attenuated, metaphysics serves a purpose in helping us grasp how the world could be the way physics says it is.

Acknowledgements I'd like to thank the two referees for detailed and helpful suggestions. I'd also like to thank for their comments and general support, Kristina Engelhard, Julia F. Göhner, Katherine Hawley, Kerry McKenzie, Juha Saatsi, Markus Schrenk, Barbara Vetter and the audience in the joint GAP-GWP Colloquium on Analytic vs Naturalised Metaphysics at the 9th German Analytic Philosophy Conference in Osnabrück in September 2015.

Open Access This article is distributed under the terms of the Creative Commons Attribution 4.0 International License (http://creativecommons.org/licenses/by/4.0/), which permits unrestricted use, distribution, and reproduction in any medium, provided you give appropriate credit to the original author(s) and the source, provide a link to the Creative Commons license, and indicate if changes were made.

\section{References}

Baker, A. (2009). Mathematical explanation in science. British Journal for the Philosophy of Science, 60(3), 611-633.

Bauer, W. A. (2011). An argument for the extrinsic grounding of mass. Erkenntnis, 74, 81-99.

Beebee, H. (2000). The nongoverning conception of laws of nature. Philosophy and Phenomenological Research, 61, 571-594.

Bird, A. (2007). Nature's metaphysics: Laws and properties. Oxford: Oxford University Press.

Bird, A. (2014). Time, chance and the necessity of everything. In A. Wilson (Ed.), Chance and temporal asymmetry (pp. 280-293). Oxford: Oxford University Press.

Black, M. (1952). The identity of indiscernibles. Mind, 61, 153-164.

Callender, C. (2011). Philosophy of Science and Metaphysics. In S. French \& J. Saatsi (Eds.), The continuum companion to the philosophy of science (pp. 33-54). London: Continuum.

Casullo, A. (1984). The contingent identity of particulars and universals. Mind, 123, 527-554.

Chakravartty, A. (2013). Realism in the desert and in the jungle: Reply to French, Ghins, and Psillos. Erkenntnis, 78, 39-58.

Chalmers, D. (2002). Does Conceivability entail possibility? In J. Hawthorne \& T. Gendler (Eds.), Conceivability and possibility (pp. 145-200). Oxford: Oxford University Press.

Eddington, A. (1941). Discussion: Group structure in physical science. Mind, 50, 268-279.

Eddington, A. (1946). Fundamental theory. Cambridge: Cambridge University Press.

French, S. (1995). Hacking away at the identity of indiscernibles. The Journal of Philosophy, 92, 455-466.

French, S. (2014). The structure of the world. Oxford: Oxford University Press.

French, S. (2018). Realism and metaphysics. In J. Saatsi (Ed.), Routledge handbook of realism. Routledge, pp. 394-406.

French, S., \& Krause, D. (2006). Identity in physics: A historical, philosophical, and formal analysis. Oxford: Oxford University Press.

French, S., \& McKenzie, K. (2012). Thinking outside the (tool)box: Towards a more productive engagement between metaphysics and philosophy of physics. The European Journal of Analytic Philosophy, 8, 42-59.

French, S., \& McKenzie, K. (2015). Rethinking outside the toolbox: Reflecting again on the relationship between philosophy of science and metaphysics. In T. Bigaj \& C. Wuthrich (Eds.), Metaphysics in contemporary physics. Poznan Studies in the Philosophy of the Sciences and the Humanities (pp. 145-174). Amsterdam: Rodopi.

French, S., \& Redhead, M. (1988). Quantum physics and the identity of indiscernibles. British Journal for the Philosophy of Science, 39, 233-246.

French, S., \& Saatsi, J. (2018). Symmetries and explanatory dependencies. In J. Saatsi \& A. Reutlinger (Eds.), Explanation beyond causation. Oxford University Press, pp. 185-205.

French, S. (forthcoming). Doing away with dispositions. In A. Meincke (Ed.), Dispositionalism: Perspectives from metaphysics and the philosophy of science. Springer Synthese Library: Springer.

Hacking, I. (1975). The identity of indiscernibles. Journal of Philosophy, 72(9), 249-256. 
Hall, N. (2015). Humean reductionism about laws of nature. In B. Loewer \& J. Schaffer (Eds.), A companion to David Lewis. Oxford: Wiley.

Hawley, K. (2006). Science as a guide to metaphysics. Synthese, 149, 451-470.

Hawley, K. (2009). Identity and indiscernibility. Mind, 118, 101-119.

Huggett, N., \& Norton, J. (2014). Weak Discernibility for quanta: The right way. British Journal for the Philosophy of Science, 65, 39-58.

Ladyman, J., \& Bigaj, T. (2010). The principle of identity of indiscernibles and quantum mechanics. Philosophy of Science, 77, 117-136.

Ladyman, J., \& Ross, D. (2007). Every thing must go: Metaphysics naturalized. Oxford: Oxford University Press.

Lange, M. (2012). There sweep great general principles which all the laws seem to follow. In K. Bennett \& D. Zimmerman (Eds.), Oxford studies in metaphysics (Vol. 7, pp. 154-185). Oxford: Oxford University Press.

Lowe, E. J. (1998). The possibility of metaphysics. Oxford: Oxford University Press.

McKenzie, K. (2014). Priority and particle physics: Ontic structural realism as a fundamentality thesis. British Journal for the Philosophy of Science, 65, 353-380.

Melia, J. (2000). Weaseling away the indispensability argument. Mind, 109(435), 455-479.

Molnar, G. (1999). Are dispositions reducible? The Philosophical Quarterly, 49, 1-17.

Muller, F., \& Saunders, S. (2008). Discerning fermions. British Journal for the Philosophy of Science, 59, 499-548.

Pust, J. (2017). Intuition. In E. N. Zalta (Ed.), The stanford encyclopedia of philosophy (summer 2017 edition). https://plato.stanford.edu/archives/sum2017/entries/intuition/. Accessed July 2017

Quine, W. V. O. (1976). Grades of discriminability. The Journal of Philosophy, 73, 113-116.

Saatsi, J. (2011). The enhanced indispensability argument: Representational vs. explanatory role of mathematics in science. British Journal for the Philosophy of Science, 62, 143-154.

Saatsi, J. (2016). On explanations from geometry of motion. British Journal for the Philosophy of Science. https://doi.org/10.1093/bjps/axw007.

Saatsi, J. (2017). Explanation and explanationism in science and metaphysics. In M. Slater \& Z. Yudell (Eds.), Metaphysics and the philosophy of science: New essays. Oxford University Press, pp. 163-192.

Saunders, S. (2003). Physics and Leibniz's principles. In K. Brading \& E. Castellani (Eds.), Symmetries in physics: Philosophical reflections. Cambridge: Cambridge University Press.

Sider, T., Hawthorne, J., \& Zimmerman, D. (2007). Contemporary debates in metaphysics. Oxford: Blackwell.

Strevens, M. (2008). Depth: An account of scientific explanation. Harvard: Harvard University Press.

Vetter, B. (2009). Review of bird. Logical Analysis and History of Philosophy, 8, 320-328.

Vetter, B. (2015). Potentiality: From dispositions to modality. Oxford: Oxford University Press.

Woodward, J. (2003). Making things happen: A theory of causal explanation. Oxford: Oxford University Press.

Yablo, S. (1993). Is conceivability a guide to possibility? Philosophy and Phenomenological Research, 53, $1-42$. 